\title{
Iron restriction in renovascular hypertension
}

\author{
Hypertension Research (2017) 40, 627; doi:10.1038/hr.2017.26; published online 2 March 2017
}

In a recent issue of the Journal (Hypertension Research, Vol. 39, no. 12), Oboshi et al. ${ }^{1}$ reported the effects of iron restriction (IR) in a rat model of renovascular hypertension (RVH). Specifically, the authors reported that IR attenuated hypertension and renal damage in a two-kidney one-clip (2K1C) model of RVH. However, their model may not be appropriate for $\mathrm{RVH}$, and the compensatory hypertrophy of the contralateral kidney reported by the authors may be proportional to animal growth.

In the $2 \mathrm{~K} 1 \mathrm{C}$ model, renal artery clipping caused atrophy of the affected kidney (Figure If of the report), indicating that the clipping procedure had caused unexpected stenosis of the renal artery. This stenosis impeded renal perfusion, resulting in ischemia and subsequent atrophy of the affected kidney. With appropriate clipping, the affected kidney is not expected to atrophy in the absence of antihypertensive treatment in $\mathrm{RVH}^{2}{ }^{2}$ In $\mathrm{RVH}$, renin-angiotensin system (RAS) activation compensates for ischemia in the affected kidney by increasing systemic blood pressure (BP). As long as the compensation is appropriate, hypertension will persist and the affected kidney will not atrophy. The gradual reductions in systemic BP to nearly normal levels that were noted in the model (Figure 1a of the report)-phenomena suggestive of reduced RAS activation-indicated that the model was not appropriate for RVH.

As the authors noted, IR attenuated compensatory hypertrophy of the contralateral kidney in $2 \mathrm{~K} 1 \mathrm{C}$ animals, as the weight of the contralateral kidney was lower in animals treated with IR than those not treated with IR (Figure 2a of the report). This attenuation was believed to be proportional to the body weights (BWs) of the animals. According to the graph in Figure 2a, the weight of the contralateral kidney was $\sim 15 \%$ lower in IR-treated animals than in non-treated animals. Moreover, the BWs of the IR-treated group were $\sim 15 \%$ lower than those of the non-treated group (Table 1 of the report). Taken together, these findings indicate that the reduction in compensatory renal hypertrophy elicited by IR was proportional to the reduction in $\mathrm{BW}$ elicited by the treatment.

IR-induced reductions in systemic BP and BW have been reported previously. ${ }^{3,4}$ In these reports, IR reduced food intake and feeding efficiency, which may have resulted in growth retardation. Reduced food intake will cause reductions in potassium intake, resulting in reduced urinary potassium excretion and reduced mineralocorticoid activity, as presented in Figure 4 of the report. ${ }^{1}$ Reduced food intake may also have contributed to the above-mentioned reductions in systemic BP in the RVH model. These findings indicate that food intake restriction causes growth retardation similar to that caused by IR, and may thus attenuate hypertension and renal damage in a manner similar to the manner in which IR attenuates such phenomena.

\section{CONFLICT OF INTEREST}

The author declares no conflict of interest.

Masayuki Tanemoto

Department of Internal Medicine, Division of Nephrology, Shin-Kuki General Hospital, Saitama, Japan E-mail: mtanemoto-tky@umin.ac.jp

1 Oboshi M, Naito Y, Sawada H, Iwasaku T, Okuhara Y, Eguchi A, Hirotani S, Mano T, Tsujino T, Masuyama T. Attenuation of hypertension and renal damage in renovascular hypertensive rats by iron restriction. Hypertens Res 2016; 39: 832-839.

2 Michel JB, Dussaule JC, Choudat L, Auzan C, Nochy D, Corvol P, Menard J. Effects of antihypertensive treatment in one-clip, two kidney hypertension in rats. Kidney Int 1986; 29: 1011-1020.

3 Strube YN, Beard JL, Ross AC. Iron deficiency and marginal vitamin A deficiency affect growth, hematological indices and the regulation of iron metabolism genes in rats. J Nutr 2002; 132: 3607-3615.

4 Turner LR, Premo DA, Gibbs BJ, Hearthway ML, Motsko M, Sappington A, Walker L, Mullendore ME, Chew HG Jr. Adaptations to iron deficiency: cardiac functional responsiveness to norepinephrine, arterial remodeling, and the effect of beta-blockade on cardiac hypertrophy. BMC Physiol 2002; 2 : 1. 